\title{
Das neue Fragment einer Sintfluterzählung und der Priesterkodex.
}

Vom Herausgeber.

Unter den nach Zehntausenden zählenden Inschriften, welche die Amerikanische Expedition der Universität von Pennsylvanien der verschütteten alten Tempelbibliothek in den Ruinenhügeln von Nippur enthoben hat, ist vor kurzem von $\mathrm{H}$. V. HILPRECHT ein Text entdeckt worden, der ein Bruchstück einer alten Sintfluterzählung enthält, welche von den übrigen bisher bekanntgewordenen babylonisch-assyrischen Versionen abweicht. HILPRECHT hat von seinem Funde eingehenden Bericht erstattet, zuerst in englischer ${ }^{x}$ und jetzt auch in deutscher ${ }^{2}$ Darlegung.

Jede neue Version der Sintfluterzählung erweckt schon an sich das Interesse, und dies um so mehr, je älter dieselbe ist. Nun soll nach HILPRECHT das neue Fragment aus dem Ende des 3. Jahrtausends (zirka aus dem. Jahre 2100 ) herrühren, also um ein beträchtliches älter sein als die beiden in 7. Jahrh. geschriebenen Nineve-Versionen, die allerdings, wie man weib, Abschriften viel älterer Dokumente darstellen. Dazu kommt aber noch, $\mathrm{da} @$ diese neugefundene Nippur-Version nach HILPRECHT zu einer der beiden alttestamentiichen Versionen in besonders naher Beziehung. stehen soll. Denn HILPRECHT konstatiert unter der Überschrift „Ergebnisse“ unter andrem S. 56 (engl. Ausg. S. 59), „daß die Übereinstimmung, die zweifellos zwischen Nippur- und Bibelversion vorliegt, sich auf denjenigen Bestandteil des Pentateuchs (Gen $6_{13-20} 7$ Ir) erstreckt, den die alttestamentliche Kritik mit P. (Priesterkodex) bezeichnet und als ,um 500 v. Chr. in Babylonien abgefabt" anzusehen pflegt". Diese Aufstellung, die wichtige Folgerungen nahelegt, muß das höchste Interesse der alttestamentlichen Wissenschaft hervorrufen; darum ist es geboten, sofort dem neuen Funde alle Aufmerksamkeit zu schenken, also aus der

I The Earliest Version of the Babylonian Deluge Story and the Temple Library -of Nippur. Philadelphia 1910. Die Schrift bildet in der großen Publikation The Babylonian Expedition of the University of Pennsylvania den Fasc. I von Bd. V der Serie D: Researches and Treatises.

2 Der neue Fund zur.Sintflutgeschichte aus der Tempelbibliothek von Nippur. Leipzig 1910. 
Zurückhaltung herauszutreten, die sonst bei solchen neuen Entdeckungen dem Alttestamentler solange ziemt, bis die Assyriologen von Fach unter sich über dieselben einigermaßen einig geworden sind. Denn auch bei dem neuen Fragment bestehen noch manche offene Fragen, die noch gelöst werden müssen und deren Beantwortung vielleicht dem Fragment ein ganz anderes Gesicht verleiht. Auch wenn die Lesung HILPRECHTs sich als im großen Ganzen richtig erweist, so ist damit noch nicht gesagt, daß alle seine Ergänzungen der Lücken auf rechter Fährte sind ${ }^{x}$ und daß wirklich, wie auch HOMMEL ${ }^{2}$ annimmt, das neue Bruchstück wie die beiden biblischen Versionen die Herbeiführung der Flut und die Rettung aus der Flut auf dieselbe Gottheit zurückführt. Ebenso ist die Herkunft des Fragments aus dem 3. Jahrtausend noch nicht über jeden $Z_{\text {weifel }}$ erhaben, da namhafte Assyriologen und Forscher, wie A. T. ClAY3, Theophilus G. Pinches 4, George A. Barton 5, geneigt sind, eher an die Kassitische Periode im 2. Jahrtausend zu denken. Aber, wie gesagt, wir müssen hier eine Ausnahme machen und dürfen nicht warten, bis die Assyriologen alle diese Fragen gelöst haben.

Das aus 14 Zeilen, wovon die erste und die letzte Zeile aber als bis auf ganz minime Reste abgebrochen nicht in Betracht kommen, bestehende Fragment lautet in der von HILPRECHT S. $60-62$ gegebenen Übersetzung folgendermaßen:

Z. 2:......., ,ich will lösen"

Z. $3: \ldots \ldots \ldots \ldots$,soll vertilgen alle Menschen miteinander";

Z. $4: \ldots \ldots$ „Leben (?), bevor die Flut hereinbricht",

Z. $5: \ldots \ldots$ über] ,so viele ihrer sind, will ich bringen Vernichtung,

Z. 6: ....., ,baue ein gro@es Schiff und“"

Zerstörung, Zermalmung".

Z. $7: \ldots \ldots$,gesamte Höhe soll sein sein Bau",

Z. 8: . . . ,es soll sein ein Hausboot, tragend was gerettet wird von Leben“.

Z. 9: .... ,mit einem starken Dach bedache es,"

Z. IO: [.... soll sein das Boot], „das Du bauen sollst".

Z. II : ..... „die Tiere des Feldes, die Vögel des Himmels."

Z: 12: ........, anstatt einer Anzahl";

Z. I $3: \ldots \ldots \ldots$, und die Familie"....

Man wird - immer die Richtigkeit der Übersetzung vorausgesetzt —

I Vgl. Joins Dyneley Prince and Frederick A. Vanderburgh, The New Hilprecht Deluge Tablet in AJSL XXVI, No. 4 (Juli 1910), S. 303-308.

2 S. ET XXI, No. 8 (Mai 1910), S. $369 . \quad 3$ S. ET 2aO. S. 367.

+ 5. ET 22O. S. 368 . 5 S. ET XXI, No. 10 (Aug. 1910), S. 504-507. 
zugeben müssen, daß dieser Nippur-Text von einer groljen Flut und zwar nicht bloß von einer außerordentlichen Flußüberschwemmung, sondern von der sogenannten Sintflut handelt. Die Vernichtung aller Menschen miteinander und der Bau eines grolien Schiffes zur Aufnahme der zu rettenden Lebewesen reden doch eine $z u$ deutliche Sprache. Dagegen ist es schwer einzusehen, warum diesem fragmentarischen Texte eine größere Übereinstimmung mit der Version des Priesterkodex als derjenigen des Jahwisten zugeschrieben wird, auch wenn man sich sofort daran erinnert, daß der Auftrag Elohims an Noah, ein Schiff zu bauen, in der Genesis nur in der Version des Priesterkodex wiedergegeben ist. Es ist doch kein Zweifel, daß der Jahwist diesen Auftrag in seiner Darstellung auch enthalten haben mußte und daß seine Version nur zugunsten derjenigen von $\mathrm{PC}$ vom Redaktor ausgelassen wurde. Von den charakteristischen Eigentümlichkeiten des $\mathrm{PC}$ - und diese müßten den Ausschlag geben - entdeckt man aber jedenfalls bei der ersten Betrachtung dieser neuen Version, sowie sie vorliegt, nichts. Etwas anders lautet das Urteil erst, wenn man die von HilpRECHT vorgeschlagenen Ergänzungen mit beachtet. Aber die große Frage bleibt eben die, ob diese Ausfüllungen der Lücken berechtigt sind oder nicht.

Doch HILPRECHT findet bereits im unergänzten Texte Gründe für seine Behauptung der Übereinstimmung der Nippur-Version mit $P$, und diese sind darum zuerst $z \mathrm{u}$ prüfen. HILPRECHT stellt auf S. $53 \mathrm{ff}$,, wo er die ,wesentlichsten Berührungspunkte zwischen $\mathrm{P}$ und der NippurVersion“ aufzählt, voran: „Die in Z. 6-1o der Nippur-Version gegebene Reihenfolge einzelner Instruktionen ist, soweit erhalten, genau dieselbe als in Gen $6{ }_{4}-16(P)$. Das kann von keiner andern Version behauptet werden". Aber ein Vergleich mit der jahwistischen Version ist ja ausgeschlossen, weil, wie angegeben, dieser Teil derselben fehlt. Und dann ist die Reihenfolge doch rein die natïliche, so daß ein irgendwie sicherer Schluß auf näheren literarischen Zusammenhang darauf nicht zu bauen ist. Zudem ist doch zu erwähnen, $\mathrm{da} ß \mathrm{Z} .8$ in $\mathrm{PC}$ keine Parallele hat, da die Reihenfolge in PC (Gen 6 15 16) nur für Z. 7 und 9 in Betracht kommt, und daß auch $Z$. Io $z u$ fragmentarisch erhalten ist und zu viele Deutungen zuläßt, als dabs sie mit dem Schlub von Gen 6 16 gleichgesetzt werden dürfte. Die $\cdots$ Übereinstimmung in der Reihenfolge geht aber überhaupt in die. Brüche, wenn man nicht nur Z. 6-10, sondern das ganze Fragment ins Auge faßt. Nach HILPRECHTs eigener Aufstellung (S. 60-62) entspricht. dem Inhalte desselben PC in der Reihenfolge: Gen 7 I1 6 I3 18 17 14 15 16 19.20 $18^{b}$. 
Ähnliches gilt von dem zweiten Berührungspunkte, den HILPRECHT anführt: „Unter den in Z. 7 erhaltenen Bezeichnungen für die Dimensionen der Arche steht die ,Höhe' an letzter Stelle, genau wie in P., Gen $615^{\prime \prime}$. Denn daß man bei den Anleitungen zu der Errichtung eines Baues die Höhe zuletzt angibt, ist ganz natürlich, und wiederum fehlt der Paralleltext des Jahwisten zu einer Vergleichung.

Auch die weitere Parallele, daß dás Fragment Z. 9 und der Priesterkodex Gen 6 i6 die Anfertigung eines Daches gebieten, kann irgend etwas für die nähere Verwandtschaft der Nippur-Version und des PC nicht beweisen. Auch der Jahwist kennt eine Bedachung der Arche, s. Gen 8 13, und daß der Befehl an beiden Orten, im Fragment und im PC, ,ähnlich einfach, rein sachlich" gehalten ist, kann doch nur natürlich erscheinen. $\mathrm{Da}$ übrigens das Fragment die längst von verschiedenen Alttestamentlern dem "Sָ gegebene Bedeutung "Dach" empfiehlt, ist bereitwilligst anzuerkennen.

Wenn ferner darauf hingewiesen wird, daß beide Versionen die Vögel unter den Tieren der Arche erwähnen, so soll das wohl auch nach HILPRECHT nicht entscheidend sein, da er in Klammern anmerkt, daß sie sich auch beim Jahwisten finden. Ebenso wird er dem Umstand kein großes Gewicht beimessen, daß die Nippur-Version in Z. 5 und der PC in 613 und 617 zur Bezeichnung der von der Flut angerichteten Vernichtung sich einer. Häufung von Synonymen bedienen.

Das Hauptgewicht der Beweisführung HILPRECHTs ruht schließlich darauf, daß er in der Nippur-Version das für den PC charakteristische Wort $m \hat{\imath} n$ in demselben Zusammenhange Z. 12 und PC Gen 620 findet. Er liest nämlich Z. 12 ku-um mi-ni, das er mit anstatt einer Anzahl

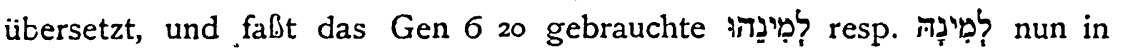
der Bedeutung: statt einer Anzahl davon. Wäre das alles richtig, so mübte eine gewisse Verwandtschaft des Nippur-Fragments mit PC zugegeben werden, nämlich der Gebrauch des für PC charakteristischen Wortes I9 2229 nur noch Dtn $14{ }_{13}-1518$ Hes 47 ro im AT vorkommt, sich aber auch in JSir 13.15f. 4325 und dann häufig im späteren Hebräisch, im Aramäischen und Syrischen findet, also der späteren hebräischen Literatur angehört. 'Aber in dieser Aufstellung HILPRECHTs ist nun alles fraglich, ja was in Bezug auf das AT gesagt ist, entschieden unrichtig. Es

I Geazuer wäre zu sagen: „Die Angabe über die ,Höhe' der Arche steht am Schlusse, wie in Gen $6 x_{5}$ ". Andere „Bezeichnungen für die Dimensionen“ sind ja nicht erhalten. 


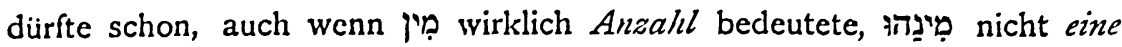
Allaahl davon übersetzt werden, sondern nur scine Anzahl. Dann ist es trotz aller Miihe, die sich HiLPREchT mit Heranziehung von Beispielen, wie fïr jedcn Stamm gibt, nicht berechtigt, ?, hier im Unterschied von Gen I, die Bedeutung von anstatt zuzuschreiben. Endlich aber ist sehr viel verlangt, wenn statt der in den Zusammenhang durchaus passenden Bedeutung Art dem pạ die sehr fragliche Bedeutung Zahl gegeben werden soll. Man bedenke doch, wie nichtssagend es wäre, wenn Gen 620 verstanden werden wollte: Von den Vögeln statt einer Zahl davon (oder statt ihrer Zahl) sollen zwei zu dir in die Arche eingehen, damit sie an Leben bleiben, gegenüber dem Sinn, den die Stelle wirklich hat: Von den Vögeln je nach ilurer Art sollen zwei zu dir in die Arche eingehen..... Alle Vogelarten sollen erhalten bleiben, nicht nur zwei Vertreter der ganzen Vogelwelt., Und was die Etymologie von מִ betrifft, so ist es viel natürlicher an einen Stamm מין zu denken als

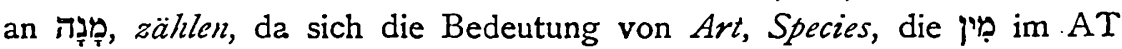
hat, und von Sekticrer, den es später bezeichnet, nicht von $z \ddot{n}$, sondern sehr wohl von dem im Arabischen erhaltenen Verbum (هيب) مان furchen, spalten (vgl. مان الارض اذا شقّها للزراعة) als eine Abspaltung herleiten läßt. Ist somit die Behandlung der alttestamentlichen Parallele für Z. 12 sehr zu beanstanden, so ist selbst der Text der keilschriftlichen Stelle nicht sicher und seine Übersetzung noch weniger. Es ist nicht gewi $\emptyset$, daß mit HILPRECHT in $k u-\imath m m$ mi-ni abzutrennen ist (- andere Assyriologen machen andere Vorschläge -), und demgemäß bleibt die Übersetzung anstatt einer Anzahl noch zweifelhafter.

Nimmt. man auch als wahrscheinlich an, daß mi-ni zu lesen sei, so beruht die nähere Beziehung der Nippur-Version zu dem Priesterkodex lediglich auf dem Gleichklang von mi-ni und jִ̣̆, der aber auf alle Fälle viel zu schwach ist, um die These HILPRECHTs zu tragen. Erst die Ergänzungen der Lücken, namentlich der Lücke in dieser Zeile I2, durch HiLPRECHT ändern die Sachlage. Darum kommt alles darauf an, ob diese Ergänzungen so, wie sie HILPRECHT gibt, notwendig sind. $\mathrm{Zu}$ Z. 2 ,ich will lösen" ergänzt er als Objekt‘, die Grenzen von Himmel und Erde", was (wie HILPRECHT selbst erwähnt) an die Vorstellung von PC erinnern würde', daß bei der Sintflut die Quellen des Ozeans aufbrachen und die Fenster des Himmels sich auftaten (Gen 7 II). Aber eine Nötigung zu dieser Ergänzung liegt nicht vor, am allerwenigsten in dieser speziellen Form. $\mathrm{Zu}$ dem Verbum „ich will lösẹn." könnte 
auch ganz anderes Objekt sein. Ebenso sind die Ergänzungen $z u$ den folgenden Zeilen, die dem Sinn nach das Richtige treffen mögen, nicht entscheidend. In Z. 3 wird "einen Flutstrom will ich machen und er" gesetzt vor "soll vertilgen alle Menschen miteinander", in Z. 4: "aber du suche $L(e b e n)$ " vor ",(L)eben, bevor die Flut hereinbricht", und in Z. 5 ",denn ïber alle lebenden Wesen" vor ,so vicle ilurer sind". Fraglicher mag die Ergänzung des Verbums "in dasselbe bringe" in Z. I I vor "die Tiere des Feldes, dic Vögel des Himmels" sein. Nun aber bringt die Ergänzung zu Z. 12 ein Charakteristikum von PC herein, wenn hier ,"und das Gewuirm, zzvei von jedem" dem "anstatt einer An$z a h l$ " vorgesetzt wird; denn der Priesterkodex macht bekanntlich in der Sintfluterzählung keinen Unterschied zwischen reinen und unreinen Tieren und läßt von allen Arten je zwei in die Arche hineingehen. Hilprecht hält sich hierzu für berechtigt auf Grund seiner Annahme, daß im allgemeinen eine Übereinstimmung zwischen dem Fragment und PC vorliege, und auf Grund seiner Fassung von $k u t-u m m$ mi-ni $=$,anstatt einer Anzahl". Ist aber die Annahme einer Übereinstimmung, wie oben gezeigt wurde, hinfällig, so könnte auch, wenn ,anstatt einer Anzahl" als richtig betrachtet werden dürfte, die Einschiebung von „zzvei von jedem" lange noch nicht für sicher gelten: warum sollten nicht zwei Kategorien unterschieden oder eine ganz andere $Z a h l$ zu vermuten sein? Da jedoch „anstatt einer Anzahl" selber auch unsicher ist, kann am Anfang von $Z$. I2 ganz andres zu ergänzen sein und die Zeile ganz andres haben sagen wollen.

Man sieht, die neue Nippur-Version ist keineswegs geeignet, um zu einer Revision der in der kritischen Schule gewonnenen Anschauungen vom $P C$ zu veranlassen. Selbst wenn die Ergänzung der $Z$ weizahl und die Fassung von Z. 12 durch HilPRECHT zufällig das Rechte träfe, so würde das kritische Urteil über die Entstehungszeit des PC nicht umgestoßen, ist es doch längst bekannt, daß der gelehrte Verfasser des PC auch altes Material verwertete, trotzdem seine Anschauung vom Verlauf der alten Geschichte und von der stufenweisen Entstehung der israelitischen Religion eine völlig neue ist.

Ist also die These von der Übereinstimmung des neuen Fundes mit dem PC und die in derselben nahegelegte Schlubfolgerung abzulehnen, so behält die glückliche Entdeckung HilPRECHTs einen hohen Wert: Das Nippur-Fragment gibt eine neue Version oder doch eine Variante oder mindestens, wie schon vermutet worden ist, eine abgekürzte Inhaltsangabe der altbabylonischen Sintfluterzählung. 
scheinen des grolien Apparats von WORDSWORTF-WHTE von den Tausenden, die diesen lateinischen Text benutzten, niemand ahnen konnte, dals er an einzelnen Stellen Lesarten bietet, die von keiner einzigen alten Handschrift bezeugt sind, so und noch hilfloser standen und stehen die meisten dem hebräischen Text gegenüber. Denn die Variantensammlungen von DE ROSSI und KENNIKOTT sind in den wenigsten Händen; die Ausgabe von Boothroyd (Pontefract, in 2 Bänden), die am unteren Rand die Handschriften anführt, ist bei uns so gut wie unbekannt. Niemand wußte daher bis jetzt und wei@ es auch bei Benutzung von KITTEL bei vielen Stellen nicht, wie es mit der Bezeugung des Textes steht, den er vor sich hat; das kann er aus der Benützung des GinSBURGschen Apparats lernen, auch wenn derselbe meinem Ideal und KITTELs Kritik nicht entspricht. . Aus einer Vergleichung des GinsBURGschen Apparats mit KITTELs Text kann sich jedermann überzeugen, daß KrTtel an einzelnen Steblen gegen BEN CHAJjIM Lesarten beibehalten, an andern solche aus BEN CHAJJIM aufgenommen hat, die in den von GINSBURG verglichenen Handschriften - und darunter sind die ältesten bis jetzt bekannten, und sind Handschriften der verschiedensten Schulen - so gut wie gar nicht bezeugt sind. Einen solchen Apparat nennt man dann wertlos; und umgekehrt einen solchen Text nennen sachunverständige Kritiker die einzige Ausgabe, die man jetzt benützen dürfe. Sapienti sat.

[Abgeschlossen am 5. August rgro.]

Schlußwort des Herausgebers.

Es kann nicht die Aufgabe des Herausgebers sein, den geschätzten Mitarbeitern mit Zustimmung oder Ablehnung ins Wort zu fallen, da sie ja selber für ihre Beiträge die Verantwortung zu tragen haben. Darum habe ich mich in der Kontroverse zwischen NeStLE und KITTEL (siehe S. I 53 f. und 229-239) jeder Einmischung enthalten und selbst ohne peinliche Nachprüfung ihre Beiträge aufgenommen, auch in der Darstellung jedem seine Eigenart unbeanstandet gelassen, in der zuversichtlichen Annahme, da die Leser dieser Eigenart das richtige Verständnis entgegenzubringen wissen. Trotzdem ich hoffen darf, in dieser Annahme mich nicht getäuscht $z u$ haben, muß ich jetzt, da die sachliche Kontroverse zu Ende geführt und jeder Leser über die Textausgaben von KITTEL und GINSBURG in ihrem Verhältnis zu BEN. CHAJJIM hinlänglich orientiert ist, ${ }^{\mathrm{x}}$ zu ein paar Schlu@bemerkungen das Wort ergreifen.

I Nachträglich teilt mir KITTEL noch mit: „Dem auf S. 229. Ausgeführten kann ich nunmehr, nachdem ich in den Ferien Gelegenheit hatte, mit Herrn KAHAN ein- 
Ich habe nämlich, was ich leider bei der Einsendung von KITTELs Nachschrift vom. 3. Juli igro (S. 238/239) unterließ, inzwischen den darin S. 238 erwähnten Aufsatz Nestles in The Expository Times XX (IgoS/9) S. 313-315: The New Hebrew Bible of the British and Foreign Bible Society noch einmal gelesen und sehe mich deshalb nun genötigt, zweierlei zu konstatieren, was auch nach NESTLEs kurzen Ausführungen (S. $304 \mathrm{ff}$.) nicht überflüssig sein dürfte:

I. Für den Pentateuch Ginsburgs ist die von Kittel S. 239 festgehaltene Bezeichnung GINSBURG-NESTLE, auch mit einem Fragezeichen, nicht zu rechtfertigen. Denn der soeben erwähnte Aufsatz NEstles in ET führt den strikten Nachweis, daß NESTLE bei der Ausgabe von GiNSBURGs Pentateuch in keiner Weise, auch nicht als proofreader beteiligt war. Sodann geht aus dem Aufsatz deutlich hervor, daß NESTLE sich mit der neuen Ausgabe nicht identifiziert hat, sondern sie nur, was den Text betrifft, als die typographisch schönste und, was den Apparat angeht, als in Hinsicht auf die Fülle der aus Handschriften und Versionen gesammelten Varianten bis dahin unerreicht erklärt.

2. Der dem Satz I do not even care about these minutiae S. 239 von KITTEL gegebene Sinn entspricht nicht dem Zusammenhang. Nach diesem will NESTLE vielmehr sagen, daß ihm Varianten in Akzenten und Metheg, ja selbst in Bezug auf scriptio plena et defectiva als gleichgültige minutiae erscheinen im Vergleich mit der viel wichtigeren höheren Textkritik; denn nach NesTLE beginnen, wie er im Zusammenhang fortfährt, die wirklichen Schwierigkeiten erst, wenn alle diese ,Minutien“ beachtet worden sind, und dabei wird von ihm ausdrücklich erklärt: For the student, the apparatus of Kittel and of Haupt is much more useful (s. ET S. $315^{b}$ ).

Als Resultat der Auseinandersetzungen von Nestle und KitTEL läßt sich nach allem hinstellen: Beide Ausgaben (die von KITtEL und die von GINSBURG) sind weder im Text noch im Apparat vollkommen; aber die typographische Ausstattung des Textes in der GinsburG-Bibel ist schöner, der Apparat in der KITTEL-Bibel dagegen für den Studenten viel nützlicher. Und damit dürfte die Kontroverse der beiden Freunde zu ihrem Abschluß gelangt sein.

gehende Rücksprache zu nehinen, hinzufügen, dals ich mich überzeugt habe, daf Herr K. vollen Grund hatte, seine Aufgabe so zu fassen, wie er es tat. Der von ibm eingeschlagene Weg war sogar (wie auch die notwendige Untersuchung der Beschaffenheit von $\mathfrak{B}$ gelehrt hat), ertgegen meiner früheren Auffassung der tatsächlich richtige." [Nachtrag bei der Korrektur.]

Bern, den 12. August 1910.

Karl Marti. 